\title{
Introducing Adaptive filters Based on Shadow Concept for Speech Processing
}

\author{
M. Koteswara Rao ${ }^{1}$, I. Santhi Prabha ${ }^{2}$ \\ ${ }^{1}$ Department of Electronics and Communications, Srivasavi Engineering College, Pedatadepalli, \\ Tadepalligudem, AP, India \\ ${ }^{2}$ Director empowerment of women and grievances and Professor of ECE Department, Jawaharlal Nehru \\ Technological University Kakinada (JNTUK), Kakinada, AP, India \\ Email:maram_koti@yahoo.co.in
}

\begin{abstract}
This paper presents the new approach to introducing adaptive Filter with LMS Algorithm based on Shadow concept. Which is useful for the cancellation of the noise component overlap with Speech signal in the same frequency range, but fixed LMS algorithm produces minimum convergence rate and fixed steady state error. So we presents design, implementation and performance of adaptive FIR filter, based on Shadow concept, which produces minimum mean square error compare to fixed LMS, and we also obtains denoised Speech signal at output, and also we propose to calculate SNR values of Adaptive Filter with LMS algorithm with and without Shadow concept.
\end{abstract}

Keywords: Windows, LMS algorithm, Shadow concept

\section{Adaptive Filters}

Rapid Advances in the VLSI technology and digital communications/digital signal processing has broughtmore attention to the adaptive least squares (LS) methods [1]. Many digital signal processing applications requires linear filters and adaptive techniques in signal processing and analysis [2]. The reference and error channels of active noisecontrol (ANC) systems may be saturated in real-world applicationsif the noise level exceeds the dynamic range of the electronic devices. This nonlinear saturation degrades the performance ofANC systems that use linear adaptive filters with the filtered-least-mean-square (FLMS) algorithm [3]. Adaptive filters havebeen included in the syllabus of undergraduate digital signal processing (DSP) courses [4]. The LMS algorithm has been extensively used in many applications as a consequence of its simplicity and robustness [5]. LMS based adaptive filters used in all sparse systems for noise Cancellation [6]. Adaptive algorithms are applicable to system identification and modeling, noise and interference cancelling, equalization, signal detection and prediction. [7] LMS Algorithm is widely usedin a variety of applications, ranging from speech enhancement and biomedical signal processing to active control ofsound and vibration [8]. Adaptive Filters are widely used in numerous industrial applications. Accoustics, communications, automatic control and seismology [9]. Information processing in variable and noisy environmentsis usually accomplished by means of adaptive filters [10]. Adaptive filtering is frequently employed in communications, control, and many other applications in which the statistical characteristicsof the signals to be filtered are either unknown a priori or, in some cases, slowly time varying [11]. Adaptive filters provide performance excellence due to their inherent pole-zero structure as compared with adaptive finite impulse response (FIR) filters that have an all-zero form, in active noise control Application [12]. RLS Filters [13]. Adaptive Filters are highly stable and effectively attenuate and often cancel destitutions [14]. An Adaptive filters are successfully used in biomedical processing systems like Denoising of ECG Waveforms [15] Adaptive filters play an important role in modern Digital signal processing products in area such as telephone echo cancellations, noise cancellation, equalization of communications channels, biomedical signal enhancement, active noise control, and adaptive control systems [16] and many Authors are worked out on FIR Filters using different Transform techniques [17] to [20] 


\section{Shadow Mechanism}

Shadow Mechanism is successfully used in improving the spectral characteristics of windows [21] Shadow based filters are used in cardiac signal processing for elimination of noises [22].

\section{Design of Adaptive Filter with Fixed LMS Algorithm}

The Figure 1 shows the block diagram of Adaptive filter with Fixed LMS Algorithm. Which processes the noised speech signal through it. Where

$\mathrm{s}(\mathrm{n})=$ clean speech signal

$\mathrm{v}(\mathrm{n})=$ noise signal

$\mathrm{h}=$ Low pass FIR Filter

$v 1(n)=h * v(n)$

$\mathrm{d}(\mathrm{n})=$ noised speech signal, $[\mathrm{s}(\mathrm{n})+\mathrm{v} 1(\mathrm{n})]$

$y(n)=$ Filtered Noise signal

$\mathrm{e}(\mathrm{n})=\mathrm{d}(\mathrm{n})-\mathrm{y}(\mathrm{n})$, [Original speech signal]

the adjustable weights are typically determined by the LMS Algorithm, the weight update equation is

$w(n+1)=w(n)+\mu^{*} e(n)^{*} v 1(n)$

$y(n)=w(n)+e(n)^{*} v 1(n)$

\section{Steps to design adaptive Filter with Fixed LMS}

1. Create or record actual speech signal.

2. Create or record a noise signal.

3. Correlate noise by passing through a low pass filter.

4. Merge Noise signal with actual Noise signal.

5. Pass this merged signal to Adaptive filter using Fixed LMS Algorithm.

6. Calculate error $\mathrm{e}(\mathrm{n})$

7. Update weight equation $w(n)$

8. Repeat step 7 and calculate adaptive output $y(n)$ until error is minimized.

9. Calculate input SNR and output SNR

\section{Design of Adaptive Filter with Fixed LMS Algorithm based on Shadow Concept}

The Figure 2 shows the block diagram of Adaptive filter with Fixed LMS Algorithm with Shadow concept In shadow filter mechanism the Low pass filter output is feedback either positively or negatively by a shadow filter of same type or different type. Here we used the shadow mechanism to find best combination for different values of ' $\beta$ '. Hence we can derive expression of the transfer function for the shadow mechanism with positive feedback connection is,

$$
\begin{aligned}
& \bar{h}(n)=\frac{\text { Low pass Filter }}{1-(\beta * \text { Low pass Filter })} \\
& \overline{\mathrm{h}}(\mathrm{n})=\frac{\mathrm{h}}{1-(\beta * \mathrm{~h})}, \quad 0 \leq \beta \leq 1 \\
& \mathrm{v} 1(\mathrm{n})=\mathrm{v}(\mathrm{n})^{*} \bar{h}(n) \\
& d(n)=\text { noised speech signal, [s(n)+v1(n)] }
\end{aligned}
$$




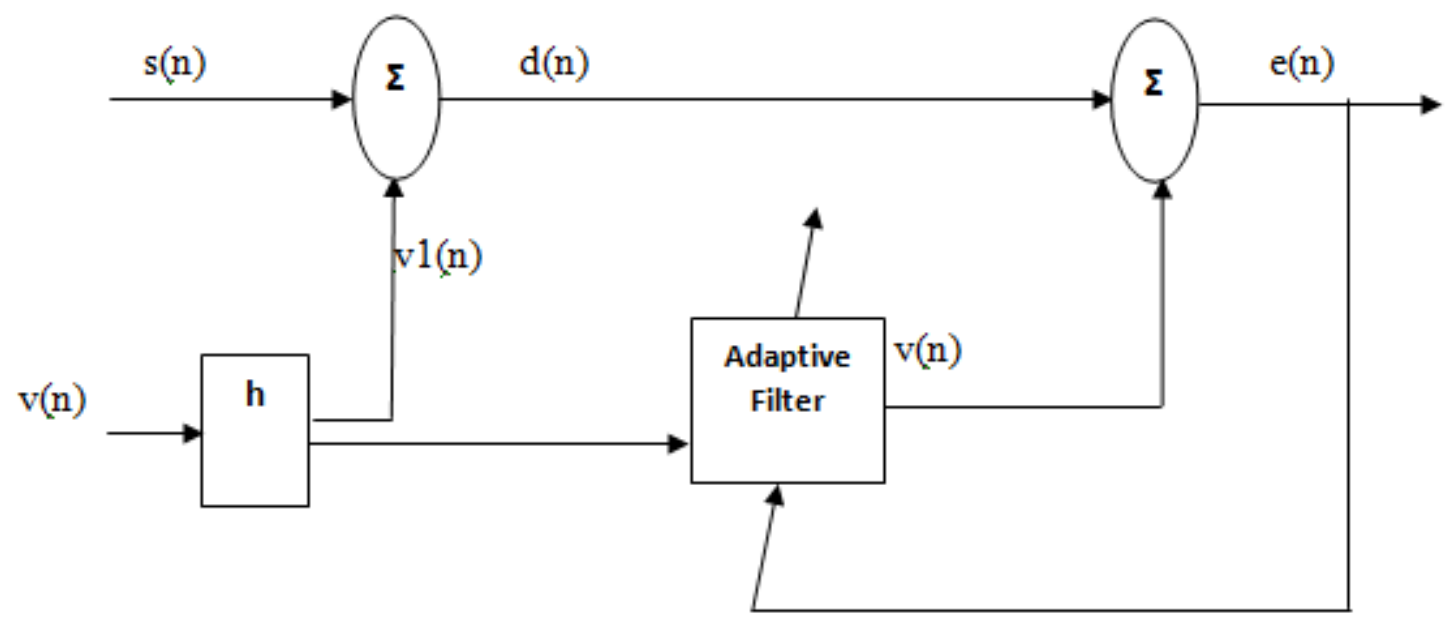

Figure 1. Block diagram of Fixed LMS Adaptive Filter

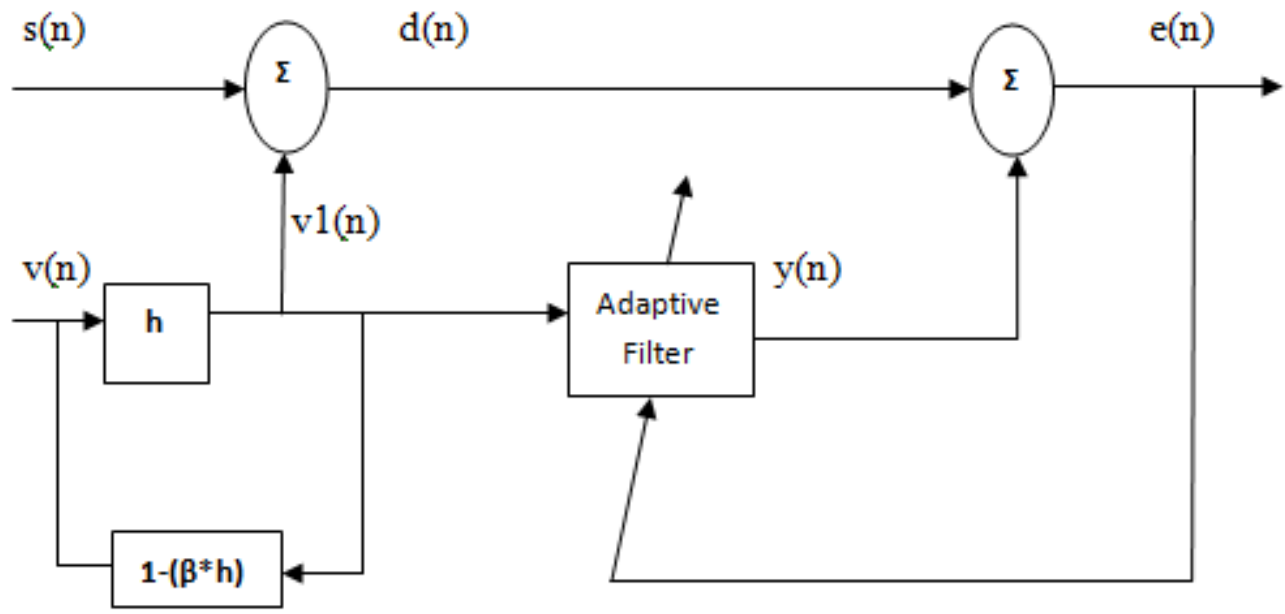

Figure 2. Block diagram of Shadow based LMS Adaptive Filter

\section{Results and Implementations}

The results shows responses of the Adaptive filter with LMS Algorithm, and we applied a noise signal to Speech and compares the signal to noise ratio of Noised signal before and after the filtering for kaiser window, which is shown in Figure 3. 

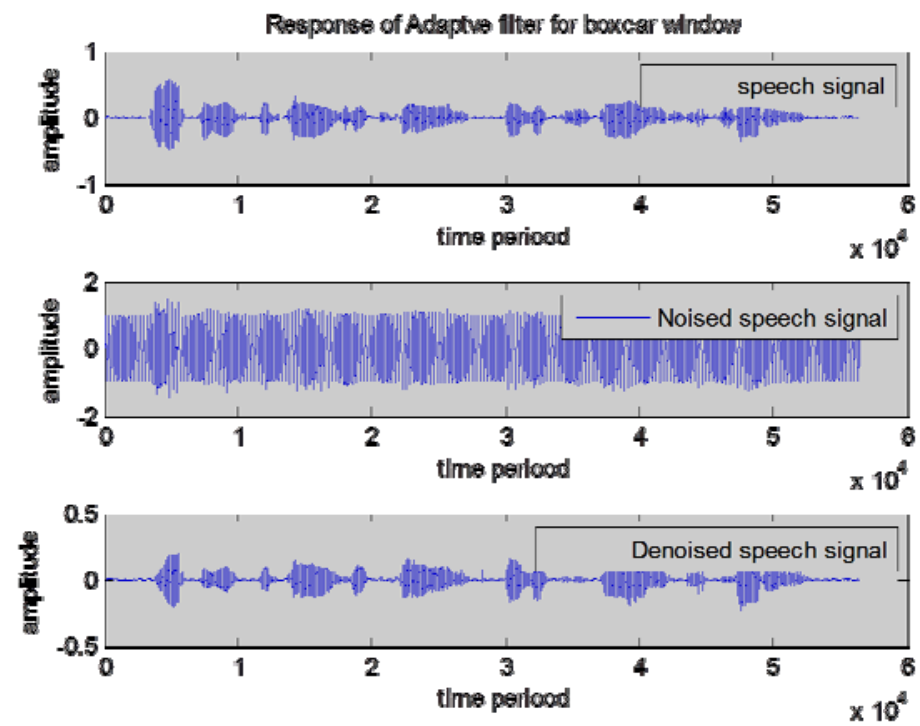

Figure 3. Denoised speech with kaiser window

When the Noised speechis filtered with Adaptive Filter with Fixed LMS algorithm the whole noise was removed, producing a near clean signal of Figure 4 to Figure 6 with different ' $\beta$ ' values of shadow FIR Filter for Kiaser window. SNR, Steady state error arecomputed for adaptive filter based on without shadow and with shadow concept are shown in Table 1, Table 2 Respectively.
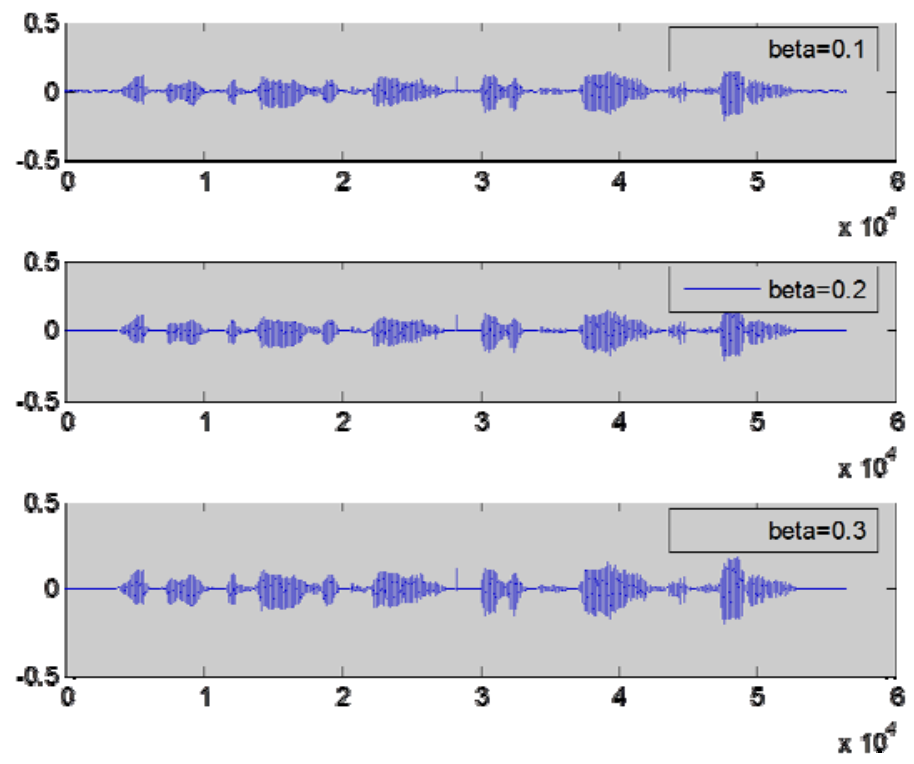

Figure 4. Denoised speech for $\beta=0.1 ; 0.2 ; 0.3$ 

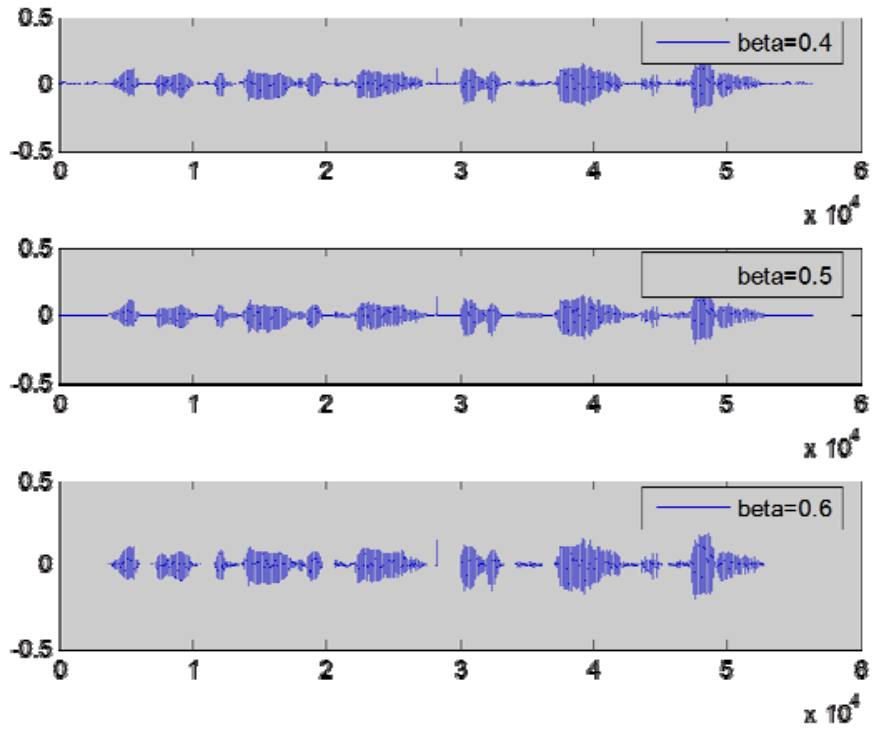

Figure 5. Denoised speech for $\beta=0.4 ; 0.5 ; 0.6$
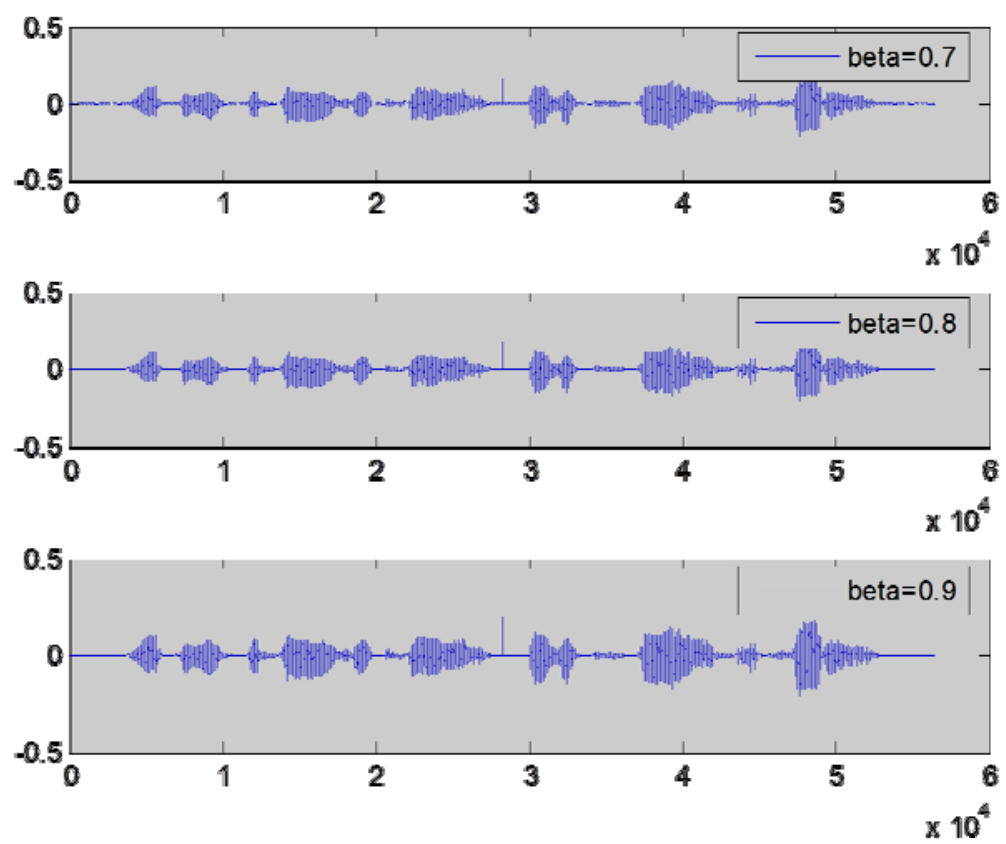

Figure 6. Denoised speech for $\beta=0.7 ; 0.8 ; 0.9$

Table1. Comparision of SNR of before and after filtering of speech signal and MSE

\begin{tabular}{ccccc}
\hline S.NO & WINDOW & $\begin{array}{c}\text { SNR before } \\
\text { Filtering in } \\
\mathrm{dB}\end{array}$ & $\begin{array}{c}\text { SNR after } \\
\text { Filtering in } \\
\mathrm{dB}\end{array}$ & $\begin{array}{c}\text { MSE } \\
\text { (Mean Square } \\
\text { Error) }\end{array}$ \\
\hline 1 & Kaiser & 0.0020 & 0.0549 & $1.5176 \mathrm{e}-012$ \\
\hline
\end{tabular}


Table 2. Comparision of SNR and MSE for Kaiser window and shadow factors

\begin{tabular}{|c|c|c|c|c|}
\hline S.NO & WINDOW & $\beta$ & $\begin{array}{c}\text { SNR after } \\
\text { Filtering in } \\
\text { dB }\end{array}$ & $\begin{array}{c}\text { MSE } \\
\text { (Mean Square } \\
\text { Error) }\end{array}$ \\
\hline 1 & & 0.1 & 0.0582 & $1.2204 \mathrm{e}-012$ \\
\hline 2 & & 0.2 & 0.0614 & $1.0022 \mathrm{e}-012$ \\
\hline 3 & & 0.3 & 0.0643 & $8.3850 \mathrm{e}-013$ \\
\hline 4 & & 0.4 & 0.0671 & $7.1330 \mathrm{e}-013$ \\
\hline 5 & & 0.5 & 0.0697 & $6.1598 \mathrm{e}-013$ \\
\hline 6 & Kaiser & 0.6 & 0.0720 & $5.3923 e-013$ \\
\hline 7 & & 0.7 & 0.0740 & $4.7795 \mathrm{e}-013$ \\
\hline 8 & & 0.8 & 0.0756 & $4.2850 \mathrm{e}-013$ \\
\hline 9 & & 0.9 & 0.0769 & $3.8824 \mathrm{e}-013$ \\
\hline 10 & & 1.0 & 0.0777 & $3.5522 \mathrm{e}-013$ \\
\hline
\end{tabular}

\section{Conclusion}

The Implementation of Adaptive-FIR Filter using shadow concept for Kiaser window was performed. And we also applied a noised speech signal to Adaptive filter and obtained denoised wave form at output which is shown in Figure 3. Later we shown responses of shadow based Adaptive filter from Figure 4 to Figure 6 for different shadow factors. We compared SNR, mean square error (MSE) at input and Output which are shown from Table 1, table 2 respectively. From the above discussions it is concluded that shadow based adaptive filter produces better responses in terms of SNR and MSE compared to Fixed Adaptive filter and Enhancement of speech signal from noised speech signal.

\section{References}

[1] Zhipei Chi, Jun Ma, and Keshab K. Parhi, "Hybrid Annihilation Transformation (HAT) for Pipelining QRD-Based Least-SquareAdaptive Filters". IEEE transactions on circuits and systems-ii: analog and digital signal processing. 2001; 48(7): 661.

[2] Daniel J Allred, HeejongYoo, Venkatesh Krishnan, Walter Huang, and David V. Anderson, "LMS Adaptive Filters Using Distributed Arithmetic for High Throughput". IEEE transactions on circuits and systems-i: regular papers. 2005; 52(7): 1327.

[3] Sen M Kuo, and Hsien-Tsai Wu, "Nonlinear Adaptive Bilinear Filters for Active Noise Control Systems". IEEE transactions on circuits and systems-i: regular papers, 2005; 52(3): 617.

[4] Emilio Soria, Javier Calpe, Jonathon Chambers, Marcelino Martínez, Gustavo Camps, and José David Martín Guerrero. "A Novel Approach to Introducing Adaptive FiltersBased on the LMS Algorithm and Its Variants". IEEE transactions on education. 2004; 47(1): 127.

[5] Yonggang Zhang and Jonathon A Chambers. "Convex Combination of Adaptive Filters for a Variable Tap-Length LMS Algorithm". IEEE signal processing letters. 2006; 13(10).

[6] Bijit Kumar Das and Mrityunjoy Chakraborty. "Sparse Adaptive Filtering by an Adaptive Convex Combination of the LMS and the za-Ims algorithms". IEEE transactions on circuits and systems-i: regular papers. 2014; 61(5): 1499.

[7] Márcio H. Costa, José Carlos M. Bermudez, and Neil J. Bershad. "Stochastic Analysis of the LMS Algorithm with a Saturation Nonlinearity Following the Adaptive FilterOutput". IEEE transactions on signal processing. 2001; 49(7).

[8] Boaz Rafaely and Stephen J. Elliott. "A Computationally Efficient Frequency-DomainLMS Algorithm with Constraints on theAdaptive Filter". IEEE transactions on signal processing. 2000; 48(6).

[9] Shin'ichi Koike. "Analysis of Adaptive Filters UsingNormalized Signed Regressor LMS Algorithm". IEEE transactions on signal processing. 1999; 47(10).

[10] Jerónimo Arenas-García, Vanessa Gómez-Verdejo, and Aníbal R. Figueiras-Vidal. "New Algorithms for Improved Adaptive Convex Combination of LMS Transversal Filters". IEEE transactions on instrumentation and measurement. 2005; 54(6): 2239.

[11] Y Zhou, SC Chan, and KL Ho. "A New Block-Exact Fast LMS/Newton Adaptive Filtering Algorithm". IEEE Transactions on Signal Processing. 2006; 54(1).

[12] Ibtissamconstantin, Regislengelle. "Performance analysis of kernal adaptive filters based on Ims algorithm". Procedia computer science 20, sciencdirect. 2013: 39-45.

[13] Michael A Vaudrey, William T Baumann, William R Saunders. "Stability and operating constraints of adaptive Ims-based feedback control". Automatics sciencdirect.

[14] Wang An Dong, Liulan, Weiqin. "An adaptive morphologic filter applied to ecg de-noising and extraction of $r$ peak at real-time". 2012 aasri conference on computational intelligence and bioinformatics, elsevier. 
[15] Kaibohu*, Yaxuanliu. "Adaptive noise cancellation method for fiber optic gyroscope". 2012 international workshop on information and electronics engineering (IWIEE), elsevier.

[16] PV Muralidhar, VLN Sastry D, SK Nayak. "Interpretation of dirichlet, bartlett, hanning and hamming windows using fractional fourier transform". International journal of scientific \& engineering research. 2013; 4(6).

[17] Muralidhar, PV DVLN Sastry, and SK Nayak. "Spectral analysis of shadow window-fir filters". Int. conf. on advances in communication, network, and computing. 2013.

[18] PV Muralidhar, AS Rao, SK Nayak. "Spectral interpretation of sinusoidal wave using fractional fourier transform based fir window functions". International review of computers and software 4.6. 2009.

[19] Muralidhar PV, AS Srinivasarao, dr SK Nayak. "Fractional fourier transform based fir window functions". Proceedings of IIndinterrnational conference rsps-2010 sponsored by IEEE (hyderabad section).

[20] Muralidhar PV, D Natraj, V Lokesh Raju and SK Nayak. "Implementation of different FIR high pass filters using Fractional Kaiser window”. IEEE International Conference. 2010; 2: 651.

[21] PV Muralidhar, DVLN Sastry and SK Naik. "Spectral Analysis of Shadow Window-FIR Filters". Proc. of Int. Conf. on Advances in Communication, Network, and Computing. 2013.

[22] N Mohana Rao, AS Srinivasa Rao, PV Muralidhar, Venkata LN Sastry D. "Noise Removal in Cardiac Signal by Shadow Digital Filters". International Journal of Engineering Research \& Technology (IJERT). 2013; 2(10), issn: 2278-0181 